\title{
La efectividad de un modelo metodológico mixto para la enseñanza-aprendizaje de español como lengua extranjera*
}

\author{
Kerwin Anthony Livingstone ${ }^{* *}$ \\ Universidad de Guyana, Guyana \\ Anita Ferreira \\ Universidad de Concepción, Chile
}

\begin{abstract}
Resumen
El presente artículo propone proveer un modelo metodológico mixto para el desarrollo e implementación de un Módulo de Enseñanza para el español como lengua extranjera en la modalidad presencial. El objetivo principal es evidenciar cómo los principios metodológicos provenientes de los enfoques didácticos ("enfoque por tareas" y "aprendizaje cooperativo") pueden ser aplicados de manera efectiva en el diseño de contextos presenciales. Para ello, se explora evidencia empírica acerca de la efectividad de la metodología mixta en la enseñanza-aprendizaje de español como lengua extranjera en dichos ambientes, en un estudio basado en un diseño experimental
\end{abstract}

${ }^{*}$ La investigación que se presenta en este artículo se circunscribe al contexto del Proyecto FONDECYT 1080165.

${ }^{* *}$ Para correspondencia dirigirse a: Kerwin Anthony Livingstone (kerwinlivingstone@ yahoo.com), Departamento de Lenguas y Estudios Culturales, Escuela de Educación y Humanidades, Universidad de Guyana, Turkeyen, East Coast Demerara, Guyana, América de Sur. 
longitudinal con pre-test y post-test, sin grupo control. Los hallazgos les proporcionarán guías de orientación efectiva a investigadores, educadores y profesores de lenguas en función del desarrollo de unidades de trabajo y módulos para el aprendizaje de lenguas extranjeras. Los resultados muestran un incremento en el aprendizaje de determinados conocimientos en español como LE y, por tanto, mejorando la competencia lingüística y comunicativa de los sujetos. Se propone, entonces, que en el diseño de módulos de enseñanza para el aprendizaje de lenguas se integren e implementen modelos metodológicos mixtos por cuanto son los más propicios para el aprendizaje de LE/L2.

Palabras clave: enseñanza-aprendizaje de lenguas mediante tareas, el enfoque por tareas, enfoque cooperativo, aprendizaje cooperativo, modelo metodológico mixto.

\title{
THE EFFECTIVENESS OF A MIXED METHODOLOGICAL MODEL FOR THE TEACHING AND LEARNING OF SPANISH AS A FOREIGN LANGUAGE
}

\begin{abstract}
This preset article seeks to provide a mixed methodological model for the development and implementation of a Teaching Module for Spanish as a Foreign Language in the face to face environment. The primary objective is to portray how methodological principles from different language teaching approaches (such as task based language teaching and cooperative learning) can be combined effectively in designing activities for face to face contexts. In this regard, empirical evidence is analysed in order to determine the effectiveness of the mixed methodology in the teaching-learning of Spanish as a Foreign Language in the said settings, in a study based on a longitudinal experimental design with pre-test and post-test, but without control group. The findings will help to provide researchers, educators and language teachers with valid guidelines as it relates to the development of Teaching Modules and Units for Foreign Language Learning. The results show an increase in the acquisition of specific knowledge in Spanish as a Foreign Language, thus improving the students' linguistic and communicative competence. It is therefore proposed that mixed methodological models be integrated and implemented when designing Teaching Modules for Language Learning, since they are the most suited for Second and Foreign Language Acquisition.
\end{abstract}


Key words: task-Based language learning and teaching, task-based approach, cooperative approach, cooperative learning, mixed methodological model.

Recibido: 01/08/09. Aceptado: 16/11/09.

\section{INTRODUCCIÓN}

En el ámbito de la enseñanza-aprendizaje de lenguas, hoy en día, una de las principales preocupaciones de investigadores, educadores y profesores gira en torno a la metodología de enseñanza que se está implementando, o la que se debería utilizar, para ejecutar las unidades de trabajo en el aula para enseñar español como LE. Todos ellos concuerdan en que los procedimientos metodológicos que se implementen deberían potenciar a los alumnos a mejorar sustantivamente su competencia lingüística y comunicativa en el idioma determinado. Referente a esto, los profesores de lenguas deberían actualizar y mejorar sus prácticas docentes -las cuales influirían de una manera u otra en el proceso de aprendizaje de sus estudiantes- por experimentar con las nuevas metodologías y enfoques didácticos del español como LE, tales como el Enfoque Comunicativo, Enfoque por Tareas, Enfoque Cooperativo y CALL, entre otros.

El interés por el Enfoque por Tareas y el Enfoque Cooperativo se debe al potencial que ofrecen para el diseño e implementación de cursos que respondan a las necesidades comunicativas específicas de los aprendices. En módulos didácticos, la tarea se ve como eje vertebral y primario del "input" pedagógico en la enseñanza. Se debe señalar que estos dos enfoques metodológicos tienen como objetivo fundamental capacitar al alumno para desarrollar y mejorar la competencia funcional en una lengua extranjera sin sacrificar la exactitud gramatical. Armonizan la manera en que se enseñan lenguas con lo que ha revelado la investigación de adquisición de segundas lenguas acerca de cómo se aprenden (Lee 2000; Sheen 1994; Willis 1996; Skehan 1998b). Conviene destacar también que dichos enfoques metodológicos constituyen un movimiento de evolución dentro del Enfoque Comunicativo y no resultaría tan difícil experimentar con ellos a través del diseño de módulos de enseñanza en los cuales se considere la práctica de las cuatro habilidades lingüísticas. 
El presente estudio se refiere a la efectividad de un modelo metodológico mixto -el Enfoque por Tareas y el Enfoque Cooperativo-para la enseñanzaaprendizaje de español como LE. El objetivo de este modelo es mejorar la competencia lingüística y comunicativa de estudiantes de nivel intermedio de la Universidad Estatal de St. Cloud, Minnesota, EE.UU.

Los datos para la investigación han sido recogidos a través de un estudio cuasi experimental longitudinal con pre-test/post-test, sin grupo control. La finalidad de las dos pruebas es recoger valiosa información sobre los niveles de competencia de los alumnos antes y después del proceso de intervención. Por medio de esta indagación, se pretende obtener información clara y precisa en lo que concierne a la competencia lingüístico-comunicativa de los participantes de este estudio, antes y después de implementar un módulo de enseñanza de español como LE. De igual forma, se quiere poner de relieve cuán efectivos son El Enfoque por Tareas y el Enfoque Cooperativo en el aprendizaje de determinados conocimientos.

\section{PLANTEAMIENTO DEL PROBLEMA}

En materia de enseñanza del español como LE, hoy en día, es evidente que los profesores están haciendo esfuerzos importantes para que sus alumnos sean competentes lingüística y comunicativamente. Sin embargo, los estudiantes no siempre muestran tener la competencia comunicativa para desenvolverse en la vida real fuera del aula. En efecto, su competencia en la lengua meta a veces es más lingüística que comunicativa.

En materia metodológica, a través del tiempo, han surgido diferentes enfoques para apoyar la enseñanza de la lengua (L2/LE). Uno de los primeros en desarrollarse fue el Método de Gramática-Traducción. Éste se basa en que la meta del estudio de una lengua es aprender una lengua para leer su literatura, o beneficiarse del desarrollo intelectual que resulta de este estudio.

En la metodología tradicional, se han observado algunas limitaciones en la delimitación de los procesos cognitivos involucrados en el desarrollo de la interlengua, que implican la resolución progresiva de formas y funciones y la reestructuración del conocimiento de L2 existente (Ellis 2003; Willis \& Willis 2007). Parece claro que la visión tradicional que identificaba al profesor de español como un instructor que transmitía sus conocimientos a los alumnos y que tomaba todas las decisiones relativas a la marcha de la clase, ya no resulta adecuada. 
Otro enfoque metodológico que ha surgido para apoyar la didáctica de la lengua es el Método Comunicativo. Desde el enfoque comunicativo, se plantea que el objetivo del proceso de enseñanza-aprendizaje de una LE es que los alumnos alcancen un cierto nivel de competencia comunicativa en dicha lengua. La evolución constante del enfoque comunicativo ha conducido al desarrollo de dos metodologías didácticas, la Enseñanza Mediante Tareas y el Aprendizaje Cooperativo. En un intento por convertir el aula en el escenario de procesos comunicativos reales, se propone la realización de las tareas en forma colaborativa como el eje articulador del proceso de enseñanza-aprendizaje.

Se debe señalar que hay poca evidencia de la experimentación con el Enfoque por Tareas y el Enfoque Cooperativo en la enseñanza-aprendizaje del español como LE. Se ve que a través del mundo, se están utilizando dichas metodologías didácticas para enseñar otras lenguas, tales como inglés y alemán. Este hecho obviamente pone en claro que no se han llevado a cabo muchos estudios en los cuales estos métodos han sido incorporados, a fin de determinar su efectividad en la didáctica del español. Se ha constatado que el Enfoque Comunicativo es el método que aún se está utilizando para la enseñanza de dicha lengua.

Dada la aparición de estos nuevos métodos y enfoques didácticos de la lengua en el mundo, y el creciente uso de ellos, se hace cada vez más evidente la necesidad de evaluar dichas metodologías para observar su efecto en el aprendizaje. La experiencia ha mostrado que lo que se enseñe en las clases de lenguas no es aprendido del mismo modo y en el mismo orden por los alumnos. Es evidente que existen distintos métodos pedagógicos para aprender una lengua extranjera, y que los profesores deben encontrar maneras de actualizar sus prácticas pedagógicas.

\section{ENSEÑANZA PRESENCIAL}

Esta modalidad hace referencia a dos criterios: la simultaneidad temporal de losprocesos decomunicación didácticaylapresenciafísica de formadoryalumnos en ese proceso de comunicación. Esto quiere decir que la modalidad presencial se caracteriza porque la mayoría de los procesos de enseñanzaaprendizaje coinciden en el espacio y en el tiempo. El profesor y los estudiantes comparten espacio físico en donde se dan los procesos de comunicación didáctica de manera simultánea en el tiempo. 
Nadie pone en duda que la enseñanza "cara a cara" es muy viva, cálida, humana y personal. Se podría asumir que la mejor formación posible es sin duda la formación presencial. "Quizá porque la interacción cara-a-cara entre formador y formando, entre el que enseña y el que es enseñado, es considerada uno de los factores fundamentales de todo proceso de formación" (Bartolomé 1995).

La educación presencial va acompañada de un complejo contexto que de manera informal refuerza el interés del estudiante por la actividad de aprendizaje que despliega (los compañeros, el intercambio de apuntes y puntos de vista, el repaso en equipo, las actividades extraeducativas, el contacto con el profesor; en definitiva, la comunicación interpersonal es el mejor detonante de la motivación). En general, permite implementar la interacción entre todos los integrantes de la comunidad de aprendizaje. Posibilita trabajar en grupo, lo que incrementa la motivación del estudiante. Es decir, hay una participación muy activa del alumnado.

Al utilizar este modelo se crea un aprendizaje más distribuido, es decir, las formas de entrega del conocimiento se estructuran de tal forma que cada destreza lingüística pueda obtener un desarrollo óptimo. Tanto habilidades de producción como de comprensión orales y escritas pueden verse mejoradas al ocupar una modalidad que pueda ayudar a reforzar cada una de ellas de manera más equilibrada. Asimismo, promueve el contacto más directo entre el docente y los estudiantes, el cual es vital en el proceso de enseñanzaaprendizaje de lenguas.

\section{METODOLOGÍA DE ENSEÑANZA DE LENGUAS}

El nacimiento del enfoque comunicativo, en los años 60, supuso un cambio de paradigma en la concepción de la lengua y de su enseñanza. Antes de su aparición, se concebía la lengua como un sistema de signos para transmitir ideas. Los métodos de enseñanza de lenguas extranjeras (LE) tales como el audio-lingual y el audio oral, descansaban en la enseñanza formal de la gramática y de la traducción. La corrección lingüística era lo fundamental. Éstos habían surgido como resultado directo de la necesidad de poseer un buen dominio oral y auditivo de una lengua extranjera. Se concebía la lengua como un sistema de signos para transmitir ideas. Los elementos centrales de la instrucción eran los ejercicios de repetición, ejercicios de automatismo (drills) y la creación de hábitos. 
Los defensores de esta metodología vieron la necesidad de poner énfasis en la precisión lingüística, alegando que una continuada repetición de errores llevaría a la adquisición de estructuras incorrectas y a una mala pronunciación. Las lecciones se organizaban en torno a una estructura gramatical presentada en diálogos cortos. Los alumnos solían escuchar una serie de grabaciones de conversaciones una y otra vez para luego intentar repetir con exactitud la pronunciación y las estructuras gramaticales de estos diálogos. Estas actividades suponían el servicio de habilidades receptivas (comprensión auditiva y lectora) subordinadas a las productivas (producción oral y escrita).

Los detractores del Método Audiolingual afirmaron que demasiado énfasis en la repetición y en la precisión lingüística no ayudaba a los alumnos a adquirir una competencia comunicativa en la lengua meta. Buscaron nuevas formas de presentar y organizar la enseñanza de la lengua y defendieron el Método Comunicativo como las estrategias más eficaces para enseñar una lengua extranjera.

El Enfoque Comunicativo surgió, tomando nuevas formas en tanto que se continuó reflexionando sobre la lengua, la competencia comunicativa, el aprendizaje y la adquisición de la lengua, y sobre aspectos didácticos que facilitaban este proceso. La lengua comienza a concebirse básicamente como un instrumento de comunicación y, por lo tanto, el énfasis de la enseñanza de LE pasa a ser el desarrollo de la competencia comunicativa de los alumnos. Su característica fundamental es que basa el aprendizaje de la lengua en necesidades comunicativas reales a las cuales el aprendiz de la lengua extranjera dará cumplimiento en dicha lengua. Esta nueva concepción de la enseñanza-aprendizaje de la LE recogió las aportaciones de varios campos de investigación, tales como la lingüística funcional británica (por ejemplo, J. Firth \& M. A. K. Halliday), la sociolingüística estadounidense (por ejemplo, D. Hymes, J. Gumperz \& W. Labov) y la filosofía del lenguaje o pragmalingüística (por ejemplo, J. Austin \& J. Searle).

El Enfoque Comunicativo tiene un carácter integrador pues combina el uso de las diferentes habilidades o destrezas de la lengua en función de lograr situaciones comunicativas reales y su objetivo fundamental es lograr la competencia comunicativa del estudiante. Moreno (1997: 122) sostiene que "el objetivo básico de la enseñanza de una L2 es el desarrollo de la capacidad para comunicarse en la lengua extranjera, no el dominio de las estructuras lingüísticas. El método tradicional no ponía énfasis en la producción comunicativa del estudiante, sino en el desarrollo de aptitudes como la lectura de obras clásicas".

En relación con lo anteriormente mencionado, Germany Germany \& Ferreira Cabrera (1999: 1) postulan que: "El proceso de enseñanza-aprendizaje 
de lenguas se justifica en términos de dos perspectivas educativas: la idealista y la pragmática. La visión idealista de la lengua enfatiza los aspectos afectivos del idioma, en tanto que la visión pragmática enfatiza la funcionalidad de un idioma. Por otra parte, y considerando que el estudiante debe obtener el máximo de provecho del idioma enseñado, su experiencia de aprendizaje debe estar, además, anclada en una base cultural sólida".

Durante los últimos 100 años, el supuesto de que la calidad de la enseñanza de una segunda lengua (L2) mejorará si los profesores mejoran sus formas de enseñanza ha redundado en el surgimiento de una variedad de enfoques y métodos, recursos de prácticas probadas que pueden adaptarse o implementarse, dependiendo de las necesidades contingentes (Richards \& Rodgers, 2001).

Dos de las nuevas formas que surgen del Enfoque Comunicativo son el Enfoque por Tareas y el Enfoque Cooperativo. A continuación, se discute cada uno de éstas:

\section{ENFOQUE POR TAREAS}

El Enfoque por Tareas se basa en el uso de tareas como el eje central de la planificación y la instrucción en la enseñanza de lenguas. Las tareas están propuestas como vehículos útiles para la aplicación de estos principios. La participación de los aprendices en el trabajo de tareas provee un mejor contexto para la activación de los procesos de aprendizaje que las actividades basadas en la forma. Breen (1987) define la enseñanza basada en tareas como: "Cualquier esfuerzo del aprendizaje de la lengua que tenga un objetivo particular, contenido apropiado, un procedimiento de trabajo específico, y un rango de resultados para aquellos que se encargan de la tarea".

Según Zanón (1999), se trata de organizar la enseñanza en actividades comunicativas que promuevan e integren diferentes procesos relacionados con la comunicación. Al ser reproducidos en el aula, los alumnos tienen que desplegar asimismo una serie de estrategias de uso para solucionar problemas concretos (de fluidez, de significado, etc.) en relación con la tarea propuesta. Ellis (2003: 276) sostiene que "El propósito general de la metodología basada en tareas es crear oportunidades para el aprendizaje de la lengua y el desarrollo de destrezas por medio de la construcción de conocimiento colaborativa".

De acuerdo con Estaire (2004-2005: 3), es un enfoque orientado hacia la construcción de la competencia comunicativa de los alumnos en todas sus dimensiones. Está centrado en la acción, en el desarrollo de la capacidad 
de los alumnos de "realizar cosas" a través de la lengua. Este enfoque se basa en una concepción cognitivo-constructivista del aprendizaje de LE, dentro de la cual el alumno es agente activo de su propio aprendizaje: se encuentra en un proceso continuo de construcción y reestructuración de conocimientos. Es una concepción del aprendizaje en la que cobran especial importancia: el aprendizaje significativo, el trabajo centrado en el alumno, las estrategias de aprendizaje y la autonomía de aprendizaje. Este enfoque está basado asimismo en una concepción social del aprendizaje de LE, que considera el aula como un contexto social, y que ofrece un marco rico en oportunidades para el desarrollo de la lengua.

\section{Un Marco Para La Enseñanza-Aprendizaje De Lenguas Mediante Tareas}

En la Enseñanza Basada en Tareas, las lecciones se articulan en torno a unidades didácticas centradas en un tema. Este modelo utiliza la tarea como unidad organizadora de la planificación. Es precisamente la tarea la que determina los contenidos que se deben trabajar en la unidad (Estaire \& Zanón 1990). Roca, Valcárcel \& Verdu (1990) enfatizan que "La tarea implica resolver un problema o completar una laguna de información activando un proceso mental utilizando la lengua extranjera y se considera que es a través de ese proceso mental que se internaliza o adquiere la L2. El alumno se concentra en la resolución de la tarea y "se olvida" que está en clase de L2 y, en consecuencia, aprende distraídamente, inconscientemente, jugando, pensando y/o creando". Este modelo potencia el trabajo en grupos y en pares propio del Aprendizaje Cooperativo.

Ellis (2003: 238) hace hincapié en lo que Estaire \& Zanón (1994) proponen como marco para la programación de unidades de trabajo o unidades didácticas. En éstas se disponen dos etapas: La primera fase implica una declaración general y se ocupa de estipular lo que se espera lograr mediante la unidad de trabajo. Se logra la declaración general en tres pasos realizados en este orden:

La primera fase:

1. La determinación del tema o área de interés para la unidad didáctica.

2. La planificación de la tarea final que será realizada al final de la unidad.

3. La especificación de los objetivos de la unidad de trabajo. 
La segunda fase consta de los detalles y estipula cómo se llevará a cabo la unidad didáctica. Hay tres pasos secuenciales adicionales a saber:

La segunda fase:

1. La especificación o determinación de los contenidos (temáticos y lingüísticos) necesarios para la realización de la tarea final.

2. La planificación y secuenciación de las tareas de comunicación y de apoyo lingüístico para capacitar a los alumnos a realizar la tarea final.

3. La planificación y procedimientos evaluativos a lo largo de la unidad.

El Enfoque por Tareas, en cualquiera de sus variantes, ha sido y sigue siendo el hilo conductor del quehacer didáctico de muchos educadores. Se ha considerado que la única manera de hacer un balance entre lo real y lo ideal de un marco teórico es proceder a desarrollarlo en el aula. De esta manera, los profesores y educadores podrían guiarse en las técnicas de elaboración de módulos pedagógicos para sus clases.

\section{El Enfoque Cooperativo}

A semejanza del Enfoque por Tareas, el Enfoque Cooperativo (Aprendizaje Cooperativo de Lenguas o AC) surge en el marco de la enseñanza comunicativa de idiomas en la década de los años 70 (Estaire 2004-2005). Es un enfoque de enseñanza que maximiza el uso de actividades cooperativas implicando a pares y a grupos pequeños de aprendices en el aula. Este enfoque se ha definido como "una actividad de aprendizaje en grupo que se organiza de tal manera que el aprendizaje sea dependiente del intercambio socialmente estructurado de información entre aprendices en grupos, y en el que cada aprendiz es responsable de su propio aprendizaje, y es motivado a aumentar el aprendizaje de los demás" (Olsen \& Kagan 1992: 8).

Fathman \& Kessler (1993: 128) definen el AC como "el trabajo en grupo que se estructura cuidadosamente para que todos los estudiantes interactúen, intercambien información y puedan ser evaluados de forma individual por su trabajo". Richards \& Rodgers (2001: 195) afirman que la palabra cooperativo en el AC da énfasis a otra dimensión importante: el desarrollo de aulas de lenguas que fomenten la cooperación en lugar de la competición 
en el aprendizaje. Es un enfoque diseñado para desarrollar habilidades de pensamiento crítico y la competencia comunicativa a través de actividades de interacción socialmente estructuradas.

Trujillo Sáez (2002) manifiesta que en el año 2001, el Departamento de Educación de California definió el AC de esta manera: "La mayor parte de enfoques cooperativos implican a grupos pequeños y heterogéneos, usualmente de 4 ó 5 miembros, trabajando juntos para llevar a cabo una tarea grupal, en la cual cada miembro es individualmente responsable de una parte del resultado final que no se puede completar a menos que los miembros de grupo trabajen juntos; dicho de otro modo, los miembros de grupo son positivamente interdependientes".

El AC sirve para desarrollar las competencias lingüístico-comunicativas, pero también para mejorar las competencias sociales y cognitivas, además de ser una herramienta de integración de la lengua y los contenidos curriculares, ejercicio fundamental en contextos de L2 y LE.

\section{ESTUDIO EXPERIMENTAL LONGITUDINAL}

En relación con las hipótesis de esta investigación, se llevó a cabo un estudio experimental longitudinal, con pre-test/post-test, sin grupo control, para determinar empíricamente si el modelo metodológico mixto, sustentado en la enseñanza basada en tareas y aprendizaje cooperativo, era efectivo para el aprendizaje de determinados conocimientos en español como LE. Los resultados deberían reflejar que la metodología mixta, a través de la modalidad presencial fundamentada en el enfoque por tareas y cooperativo, es efectiva para incrementar su aprendizaje en español, y por ende, optimizar sus destrezas lingüísticas y comunicativas.

Hipótesis: En el presente trabajo, las hipótesis que orientan esta investigación son:

1. Un modelo metodológico mixto, sustentado en el Enfoque por Tareas y en técnicas del Aprendizaje Cooperativo, será efectivo para el aprendizaje de determinados conocimientos en Español como LE.

2. Los estudiantes pueden incrementar su aprendizaje en español en torno a una temática determinada, y así ser más competentes lingüística y comunicativamente en aquella área. 
Objetivos: Los objetivos específicos de esta investigación son:

1. Diseñar un modelo metodológico de enseñanza-aprendizaje de español como LE basado en tareas y técnicas del Aprendizaje Cooperativo para estudiantes de nivel intermedio.

2. Verificar si el trabajo en pares y en grupos es efectivo para la realización de tareas y para el aprendizaje de español en un ámbito de conocimientos definido.

\section{METODOLOGÍA}

Muestra: Este módulo de enseñanza de español como LE fue dirigido a estudiantes de la Universidad de St. Cloud, Minnesota, que, por convenio con el Departamento de Español, vinieron durante el segundo semestre del año 2007 a cursar un conjunto de asignaturas, entre ellas, español como LE. Eran de diferentes especialidades y presentaban un nivel intermedio de español como LE, por lo que también necesitaban mejorar sus habilidades lingüísticas y comunicativas.

Selección de la muestra: Con el fin de obtener evidencia empírica del proceso de aprendizaje de español como LE y de evaluar la competencia lingüística y comunicativa en el español, se seleccionó como muestra a un grupo de 18 sujetos provenientes de la Universidad Estatal de St. Cloud, Minnesota, Estados Unidos. El promedio de edades de los participantes de la muestra fluctuaba entre los 18 y 22 . Del universo total de la muestra, $38.9 \%$ (7) eran de sexo masculino y $61.1 \%$ (11) de ellos de sexo femenino.

Los alumnos estadounidenses se hospedaron en casas de familias chilenas, con el objetivo de integrarse a la vida cotidiana de los chilenos. Estaban en Chile por medio de un intercambio estudiantil que tenía por finalidad mejorar su español como LE. La estadía consideró un semestre académico de inmersión, durante el cual se llevó a cabo el módulo de enseñanza.

Todos los estudiantes hablaban inglés como L1 y debido a que el español es un idioma cada vez más hablado en los Estados Unidos y en el mundo, se sentían impulsados a mejorarlo. Casi en la totalidad, los alumnos estudiaban español movidos por dos necesidades concretas: querían tener un mejor grado de competencia lingüística y comunicativa, y tenían interés por aprender más de la cultura y costumbre latinoamericanas. De esta manera, 
podrían regresar a su país y desenvolverse con mucha más eficacia en la comunidad hispanohablante. Los universitarios habían cursado y aprobado cuatro semestres de español en la Universidad Estatal de St. Cloud antes de poder inscribirse en el programa.

Diseño del experimento: Para diseñar los materiales y procedimientos para el modelo de aprendizaje combinado que se elaboró para este estudio, se llevó a cabo una revisión de los postulados teóricos de la metodología del Enfoque por Tareas y del Enfoque Cooperativo, y los componentes de la enseñanza presencial. Asimismo, se consideró el contexto cultural chileno para el diseño de todas las actividades que apoyaron la práctica de las cuatro habilidades lingüísticas, con el fin de facilitar el aprendizaje de la lengua. Dichas actividades servían para activar los procesos de metacongnición, reflexión, análisis y oportunidades de contacto con la lengua meta, permitiendo que el aprendizaje se internalizara y lograra de manera propicia.

Se ha considerado que la única manera de hacer un balance entre lo real y lo ideal de un marco teórico es proceder a desarrollarlo en el aula. Para ello, se diseñó y creó un módulo de enseñanza para el español como LE basado en la metodología mixta ya señalada. El objetivo era entonces probar la efectividad de este modelo metodológico mixto el cual, a la larga, podría ayudar a los profesores a adiestrarse en las técnicas de elaboración de módulos de enseñanza para sus clases.

Descripción del modelo metodológico mixto: El módulo para este estudio experimental fue diseñado siguiendo las pautas indicadas por Zanón (1999) para el Enfoque por Tareas en E/LE. Tales pautas se sustentan en el Marco de Enseñanza del Lenguaje Mediante Tareas que permite la creación de unidades didácticas en seis pasos y en el que se conjugan elementos compatibles de varias propuestas:

(1) El tema

(2) La tarea final

(3) Los objetivos

(4) Los contenidos lingüísticos y temáticos que se trabajarán a lo largo de la UD

(5) La secuenciación de tareas conducentes a la tarea final

(6) La evaluación 
En el contexto del Aprendizaje Cooperativo se delimitaron distintas técnicas que servían para apoyar la enseñanza de la lengua. Para efectos de esta investigación, las técnicas que se utilizaron en concreto fueron:

Aprendiendo Juntos (Learning Together): Su principal postulado es que debe incluir todos los principios básicos del aprendizaje cooperativo para que, de esta manera, todos los miembros del grupo logren la meta de la tarea propuesta.

Investigación en Grupo (Group Investigation): Plantea que los estudiantes organizan sus propios grupos y se dedican a estudiar un aspecto del tema propuesto para toda la clase.

Puzzle (Jigsaw): Cada miembro del grupo o cada grupo recibe una parte distinta de la información del tema específico a tratar. Luego de discutir la información que tiene cada miembro grupal o cada grupo con un grupo de "expertos", sobre la temática determinada, se reúnen para elaborar un informe. Con alumnos de los otros grupos, quienes tienen distintas partes de la información, se comienza a armar el proyecto en común.

Manuales: Se diseñaron dos manuales: un Manual de Profesor y un Manual de Apoyo para el estudiante. Éstos se describen brevemente a continuación:

Manual de Profesor: Este consiste en los seis pasos propuestos por Zanón \& Estaire (1990) bien especificados y planificados, es decir, especificación de un tema, una tarea final, objetivos, contenidos lingüísticos y temáticos, secuencia de tareas que conducen a la tarea final y procesos evaluativos. Además, cuenta con lecturas adicionales y enlaces electrónicos. Gracias a esto, el profesor de lenguas puede cumplir con su papel de guía y facilitador del alumno.

Manual de Apoyo: Este consta de una carta abierta, una introducción al módulo de enseñanza, las tareas para ejecutar, una lista de vocabulario útil en función del tema, un resumen de gramática y las mismas lecturas adicionales y enlaces electrónicos encontrados en el Manual de Profesor, por medio de los cuales el aprendiz podría interiorizarse más sobre el tema. El propósito de entregarles un Manual a los universitarios fue que tuvieran cierta autonomía y tomaran control de su propio aprendizaje, uno de los principales objetivos del Enfoque por Tareas. 
Temporalización del módulo de intervención: La temática de la unidad era "Los Hábitos Alimenticios Chilenos y Estadounidenses". Tenía una duración de 23 horas 25 minutos de clases lectivas, distribuidas en cinco semanas de clases presenciales y en dos días semanales, de dos horas cada día.

Este módulo metodológico mixto está compuesto de nueve tareas de comunicación y 7 de apoyo lingüístico. En otras palabras, éstas se elaboraron en base a objetivos gramaticales y comunicacionales claros con el propósito de ayudar a fomentar el contacto y las oportunidades de interacción en la lengua meta. Todas ellas se realizaron dentro de un período máximo de 18 horas 35 minutos. Las dos pruebas, el pre-test y el post-test, que formaron parte de la Evaluación, ocuparon las otras 4 horas 50 minutos. En la Tabla 1 , se ilustra el tiempo que dura cada tarea.

\section{Tabla 1: Período abarcado para el Módulo de intervención}

\begin{tabular}{|l|l|}
\hline \multicolumn{1}{|c|}{ Tarea } & \multicolumn{1}{c|}{ Duración } \\
\hline & 1 hora \\
\hline Pre-tarea & 2 horas \\
\hline Tarea 1 & 1 hora 40 minutos \\
\hline Tarea 2 & 2 horas \\
\hline Tarea 3 & 1 hora 10 minutos \\
\hline Tarea 4 & 3 horas \\
\hline Tarea 5 & 2 horas 45 minutos \\
\hline Tarea 6 & 3 horas \\
\hline Tarea 7 & 2 horas \\
\hline Tarea Final &
\end{tabular}

Además, es importante establecer que las tareas de focalización en la forma son entrelazadas con las tareas de comunicación, de la 1 hasta la 7, de tal forma que los aprendices puedan fijar su atención en éstas en el momento de completar la tarea comunicativa. Dicho de otro modo, las tareas 1 a la 7 van acompañadas de tareas de apoyo lingüístico. El tiempo programado para éstas incluye la realización de ambas tareas: la de comunicación y la de focalización en la forma.

Para que hubiera una organización más adecuada de cada clase, se confeccionó una planificación por lección en que se describía qué y cómo se realizaría cada sesión, incluyendo el enfoque metodológico y la vía de entrega de conocimiento (en este caso, clases presenciales). 
A continuación se presenta una tabla resumen con la especificación del número de sesiones presenciales involucradas en este modelo metodológico mixto:

Distribución del módulo de enseñanza:

\begin{tabular}{|l|l|}
\hline Clases presenciales & 18 sesiones y media \\
\hline $\begin{array}{l}\text { Evaluación (pre-test y post- } \\
\text { test) }\end{array}$ & 5 sesiones \\
\hline
\end{tabular}

\section{Clases presenciales:}

\begin{tabular}{|l|l|}
\hline $\begin{array}{l}\text { Trabajo presencial (face to } \\
\text { face) }\end{array}$ & 18 sesiones y media \\
\hline Trabajo en grupos & 16 sesiones y media \\
\hline
\end{tabular}

\section{Distribución de las habilidades lingüísticas en el módulo:}

\begin{tabular}{|l|l|}
\hline Compresión Auditiva & 17 sesiones \\
\hline Comprensión Lectora & 15 sesiones \\
\hline Producción Escrita & 17 sesiones \\
\hline Producción Oral & 17 sesiones \\
\hline
\end{tabular}

\section{Áreas de la lengua:}

\begin{tabular}{|l|l|}
\hline $\begin{array}{l}\text { Práctica de estructuras gra- } \\
\text { maticales }\end{array}$ & 15 sesiones y media \\
\hline Práctica de vocabulario & 15 sesiones y media \\
\hline
\end{tabular}

\section{Evaluación (pre-test y post-test):}

\begin{tabular}{|l|l|}
\hline Pre-test & 2 sesiones y media \\
\hline Post-test & 2 sesiones y media \\
\hline
\end{tabular}

El tiempo de duración del modelo metodológico mixto fue de 18 sesiones y media de clases presenciales y las actividades creadas para dicho modelo se realizaron durante dicho período. Las actividades estaban divididas en micro tareas, (Ellis 2003), que tenían como objetivo ayudar y guiar al alumno a llegar a la macro tarea (o tarea final). 
Implementación del módulo de enseñanza: El módulo de aprendizaje se integró en el plan de la asignatura "Costumbres y Hábitos de los Chilenos" del Departamento del Español de la Universidad de Concepción. Éste se llevó a cabo en la sala MECESUP, ubicada en el tercer piso de la Facultad de Humanidades y Arte de dicha universidad. Ésta contaba con la capacidad para los 18 sujetos.

Se decidió entregar el material particionado, a lo largo del proceso de la aplicación del módulo de enseñanza, con el objeto de cautelar que los estudiantes intentaran completar todas las tareas en casa y no participaran activamente en el desarrollo de éstas. Frente a esta probabilidad, que sin duda iba a poner en riesgo la efectividad de la metodología mixta y todo el módulo didáctico, se optó por entregarles las primeras dos tareas, es decir, la pre-tarea y la tarea 1, y luego la tarea 2, etc.

Las tareas de comunicación (pre-tarea, tareas 1-7) tanto como las de apoyo lingüístico o focalización en la forma (éstas fueron diseñadas y entrelazadas de tal forma que se realizaran inmediatamente después de llevar a cabo las de comunicación), fueron ejecutadas tal como aparecen en el Manual de Apoyo para el estudiante.

\section{ANÁLISIS Y DISCUSIÓN DE LOS RESULTADOS}

Este experimento consideró una variable independiente y una dependiente. La variable independiente se refirió a la modalidad presencial y metodología de enseñanza - enfoque por tareas y enfoque cooperativo. $\mathrm{Al}$ exponer a los alumnos a este tipo de metodología, el desarrollo de las habilidades lingüísticas del español se verá muy favorecido en contextos de enseñanza presencial. La variable dependiente correspondió al incremento en el aprendizaje que se observa en los resultados obtenidos por los participantes al comparar el pre-test con el post-test. Se aplicó la prueba estadística $t$ de Student para establecer diferencias significativas en los resultados logrados.

Es importante señalar que la estructura del pre-test y del post-test, que integra las habilidades receptivas (comprensión auditiva y lectora) y productivas (producción oral y escrita) ha sido adaptada en ciertos aspectos acorde con los objetivos generales de evaluar la competencia comunicativa general en el español. "Si los porcentajes de evaluación son de un total de $100 \%$, el $60 \%$ se asigna a la evaluación de las habilidades productivas, y el 
$40 \%$ a las habilidades receptivas" (Germany Germany \& Ferreira Cabrera 1999: 4).

La elaboración de dichas pruebas se realizó de acuerdo con los parámetros y el formato del Examen de Lenguas Modernas (MLE) ${ }^{1}$ del Consejo Examinador del Caribe $(\mathrm{CXC})^{2}$. Esta escala evaluativa es la misma que se está usando actualmente en el Caribe. Asimismo, este mismo esquema de evaluaciones se adoptó para el pre-test y el post-test aplicados. Es decir, los puntajes usados para los dos tests fueron los siguientes: la comprensión auditiva, 20 puntos; la comprensión lectora, 20 puntos; la producción oral, 30 puntos y la producción escrita, 30 puntos, dando un total de 100 puntos o $100 \%$. El pre-test constó de una prueba escrita para medir competencia lingüística y una entrevista para medir competencia comunicativa en función de los objetivos, contenidos y habilidades lingüísticas relacionados con la temática del módulo de enseñanza. El post-test era una prueba y entrevista equivalentes al pre-test, con la misma forma que midieron los mismos objetivos, contenidos y habilidades, pero con textos diferentes al pre-test para no influenciar los resultados.

El estudio cuasi-experimental longitudinal que se llevó a cabo para probar la efectividad de un modelo metodológico mixto, sustentado en la enseñanza basada en tareas y en técnicas del aprendizaje cooperativo, arrojó resultados muy favorables. Los resultados de los dos tests (pre y post) fueron analizados a través de los estadígrafos de media, mediana, moda, varianza, desviación estándar, porcentaje de variabilidad y correlación con el objetivo de describir el comportamiento de la muestra y hacer la comparación entre éstos.

En relación con las hipótesis, la Tabla 1 presenta los diferentes resultados observados en el pre-test del grupo. Éstos se ilustran para cada sujeto (S) de dicho grupo, por habilidad lingüística (acorde con el test utilizado, CXC).

Tabla 1: Resultados del pre-test (Las 4 habilidades lingüísticas)

\begin{tabular}{|c|c|c|c|c|c|}
\hline $\mathbf{S}$ & $\begin{array}{c}\text { Comprensión } \\
\text { Auditiva } \\
\mathbf{( 2 0 \% )}\end{array}$ & $\begin{array}{c}\text { Comprensión } \\
\text { Lectora } \\
\mathbf{( 2 0} \%)\end{array}$ & $\begin{array}{c}\text { Producción } \\
\text { Oral } \\
\mathbf{( 3 0 \% )}\end{array}$ & $\begin{array}{c}\text { Producción } \\
\text { Escrita } \\
\mathbf{( 3 0 \% )}\end{array}$ & $\begin{array}{c}\text { Total } \\
\mathbf{( 1 0 0 \% )}\end{array}$ \\
\hline 1 & 16 & 12,5 & 22 & 23 & 73,5 \\
\hline
\end{tabular}

1 Modern Languages Examinations.

${ }^{2}$ Entidad encargada de evaluaciones caribeñas en español como LE/L2 para hablantes de inglés y otros idiomas como L1. 


\begin{tabular}{|r|r|r|r|r|r|}
\hline 2 & 9 & 10,5 & 27 & 22 & 68,5 \\
\hline 3 & 12 & 14 & 23 & 23 & 72 \\
\hline 4 & 18,5 & 16,5 & 27 & 25,5 & 87,5 \\
\hline 5 & 13,5 & 14 & 20 & 24 & 71,5 \\
\hline 6 & 13,5 & 10,5 & 23,5 & 20 & 67,5 \\
\hline 7 & 10 & 12 & 18 & 14 & 54 \\
\hline 8 & 11 & 13,5 & 20 & 20,5 & 65 \\
\hline 9 & 16,5 & 15,5 & 28,5 & 21 & 81,5 \\
\hline 10 & 15,5 & 10,5 & 25,5 & 20 & 71,5 \\
\hline 11 & 12 & 12 & 25 & 21 & 70 \\
\hline 12 & 14,5 & 18 & 28 & 24 & 84,5 \\
\hline 13 & 11,5 & 15 & 23 & 20 & 69,5 \\
\hline 14 & 7,5 & 10,5 & 19,5 & 19 & 56,5 \\
\hline 15 & 10,5 & 15 & 23 & 21,5 & 70 \\
\hline 16 & 11,5 & 9 & 21,5 & 19,5 & 61,5 \\
\hline 17 & 11,5 & 15 & 23 & 20 & 69,5 \\
\hline 18 & 16,5 & 16,5 & 24,5 & 22 & 79,5 \\
\hline Promedio & $\mathbf{1 2 , 8 3}$ & $\mathbf{1 3 , 3 6}$ & $\mathbf{2 3 , 4 4}$ & $\mathbf{2 1 , 1 1}$ & $\mathbf{7 0 , 7 5}$ \\
\hline
\end{tabular}

Para determinar la mediana, se distribuyó a los estudiantes según el porcentaje de logro alcanzado con una amplitud de 2 . El cálculo a través de la fórmula por interpolación lineal arroja un resultado de 70. Éste significa que 9 estudiantes ( $50 \%$ de la muestra) obtuvieron un puntaje menor o igual que la mediana (54-70), en tanto que los restantes (50\% de la muestra) lograron un puntaje mayor o igual que la mediana $(70-87,5)$. En el pre-test, se puede ver que el rendimiento medio es de 70,75 puntos de un total de 100. Se observa que de un total de 18 alumnos, 10 (el 56\% de la muestra) se sitúan bajo la media obtenida.

En cuanto al rendimiento medio de la muestra por habilidad lingüística, se pueden observar los siguientes resultados en el Gráfico 1: (1) en relación con la comprensión auditiva (ítem 1), la media obtenida es de 12,83 puntos de un máximo de 20 (64\% de logro); (2) en cuanto a la comprensión lectora (ítem 2), se logra una media de 13,36 de un total de 20 puntos $(67 \%$ de logro); (3) con respecto a la producción oral (ítem 3), la media es de 23,44 de un máximo de 30 puntos ( $78 \%$ de logro) y, (4) en lo concerniente a la producción escrita (ítem 4), la media que se obtiene es de 21,11 de un total de 30 puntos ( $70 \%$ de logro). 
Gráfico 1: Rendimiento medio por habilidad lingüística en el pre-test

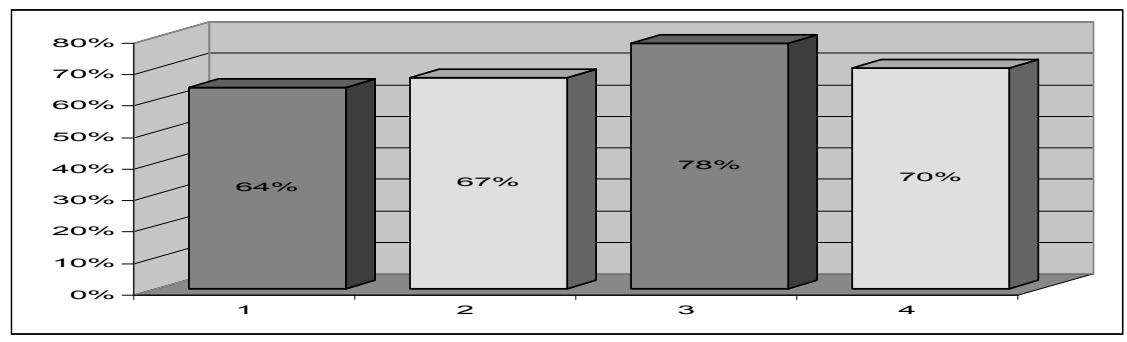

Se debe señalar que la distribución de los alumnos en rangos de porcentaje de logro en el pre-test muestra que intervalos de $61-70 \%$ y $71-80 \%$ presentan el mayor número de estudiantes. Dicho de otro modo, la mayoría de los estudiantes se situaron entre dichos intervalos. Es decir, 8 estudiantes $(44 \%$ de la muestra) tuvieron un promedio de $61-70 \%$, mientras 5 estudiantes $(28 \%$ de la muestra) obtuvieron un promedio de $71-80 \%$. Se puede resumir que 13 estudiantes ( $72 \%$ de la muestra) se encontraron entre $61-80 \%$. Los restantes ( $28 \%$ de la muestra) quedaron por debajo de este intervalo.

En relación con las hipótesis, la Tabla 2 ilustra tanto los puntajes obtenidos en el post-test por habilidad lingüística, como el puntaje total para cada uno de los estudiantes de la muestra (de acuerdo con el test utilizado, CXC).

Tabla 2: Puntaje total en el post-test (Las 4 habilidades lingüísticas)

\begin{tabular}{|r|r|r|r|r|r|}
\hline $\mathbf{S}$ & $\begin{array}{c}\text { Comprensión } \\
\text { Auditiva } \\
\mathbf{( 2 0 \% )}\end{array}$ & $\begin{array}{c}\text { Comprensión } \\
\text { Lectora } \\
\mathbf{( 2 0 \% )}\end{array}$ & $\begin{array}{c}\text { Producción } \\
\text { Oral (30\%) }\end{array}$ & $\begin{array}{c}\text { Producción } \\
\text { Escrita } \\
\mathbf{( 3 0 \% )}\end{array}$ & $\begin{array}{c}\text { Total } \\
\mathbf{( 1 0 0 \% )}\end{array}$ \\
\hline 1 & 17,5 & 17 & 27 & 26 & 87,5 \\
\hline 2 & 13 & 14,5 & 26 & 26 & 79,5 \\
\hline 3 & 12 & 8,5 & 22,5 & 24 & 67 \\
\hline 4 & 19 & 17,5 & 28,5 & 28,5 & 93,5 \\
\hline 5 & 15,5 & 15 & 27 & 25 & 82,5 \\
\hline 6 & 16,5 & 14 & 20,5 & 17 & 68 \\
\hline 7 & 9 & 11,5 & 19,5 & 17 & 57 \\
\hline 8 & 12 & 14,5 & 23 & 22,5 & 72 \\
\hline 9 & 16,5 & 18,5 & 28,5 & 22 & 85,5 \\
\hline 10 & 15 & 16 & 23 & 24,5 & 78,5 \\
\hline 11 & 17,5 & 17,5 & 25,5 & 27 & 87,5 \\
\hline 12 & 14 & 15 & 27 & 26,5 & 82,5 \\
\hline 13 & 20 & 17,5 & 26 & 26 & 89,5 \\
\hline
\end{tabular}




\begin{tabular}{|c|r|r|r|r|r|}
\hline 14 & 19 & 15 & 23 & 20 & 77 \\
\hline 15 & 17,5 & 13 & 25 & 24 & 79,5 \\
\hline 16 & 17 & 16,5 & 24 & 19 & 76,5 \\
\hline 17 & 13,5 & 15,5 & 25,5 & 24,5 & 79 \\
\hline 18 & 16 & 15,5 & 27,5 & 27 & 86 \\
\hline Promedio & $\mathbf{1 5 , 5 8}$ & $\mathbf{1 5 , 1 4}$ & $\mathbf{2 4 , 9 4}$ & $\mathbf{2 3 , 6 9}$ & $\mathbf{7 9 , 3 6}$ \\
\hline
\end{tabular}

En la determinación de la mediana del post-test, se distribuyó a los estudiantes según el porcentaje de logro obtenido con una amplitud de 8 . El cálculo a través de la fórmula por interpolación lineal arroja un resultado de 79,5. Éste indica que 9 estudiantes (50\% de la muestra) lograron un puntaje menor o igual que la mediana $(57-79,5)$, mientras que los demás estudiantes $(50 \%$ de le muestra) obtuvieron un puntaje mayor o igual que la mediana $(79,5-93,5)$. El rendimiento medio en el post-test es de 79,36 puntos de un máximo de 100. Además, se observa que de los 18 alumnos, 8 ( $44 \%$ de la muestra) quedan bajo la media. Es decir, un menor número que los presentados en el pre-test.

En lo que corresponde al rendimiento medio de la muestra por habilidad lingüística, se pueden observar los siguientes resultados en el Gráfico 2: (1) en relación con la comprensión auditiva (ítem 1), la media obtenida es de 15,58 puntos de un máximo de 20 (78\% de logro); (2) en cuanto a la comprensión lectora (ítem 2), se logra una media de 15,14 de un total de 20 puntos (76\% de logro); (3) con respecto a la producción oral (ítem 3), la media es de 24,94 de un máximo de 30 puntos ( $83 \%$ de logro) y, (4) en lo concerniente a la producción escrita (ítem 4), la media que se obtiene es de 23,69 de un total de 30 puntos ( $79 \%$ de logro).

Gráfico 2: Rendimiento medio por habilidad lingüística en el post-test

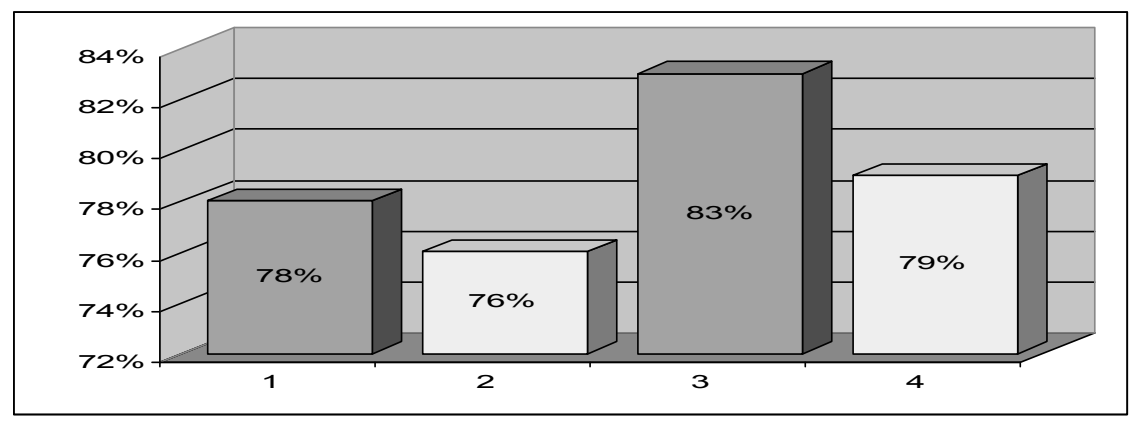


La distribución de los alumnos en rangos de porcentaje de logro en el post-test ilustra que los intervalos de $71-80 \%$ y $81-90 \%$ presentan el mayor número de estudiantes. En otras palabras, la mayoría de los alumnos se situaron entre dichos intervalos. Es decir, 7 estudiantes (39\% de la muestra) tuvieron un promedio de $71-80 \%$, mientras 7 estudiantes (39\% de la muestra) obtuvieron un promedio de $81-90 \%$. Se puede resumir que 14 estudiantes ( $78 \%$ de la muestra) se encontraron entre $71-90 \%$. Los restantes $(22 \%$ de la muestra) quedaron por debajo de este intervalo.

De los resultados encontrados en cuanto a la mediana lograda en el pretest (70\%) y la mediana alcanzada en el post-test $(79,5 \%)$ se puede apreciar un aumento en ésta de un 9,5.

En cuanto al gráfico de medias, si se contrastan los valores entre el pretest y el post-test la cantidad de alumnos que queda por debajo de éste es de 6 (33\% de la muestra). Dichos alumnos son los mismos que están bajo la media del pre-test.

En lo que corresponde al rendimiento medio logrado tanto en el pre-test como en el post-test, el Gráfico 3 muestra que la media alcanzada en el pretest es de 70,75\%, mientras la media obtenida en el post-test es de 79,36\%. Como se puede observar, los resultados encontrados, la media del post-test $(79,36 \%)$ supera a la del pre-test $(70,75 \%)$ en un $8,61 \%$.

Gráfico 3: Medias alcanzadas en el pre-test y el post-test

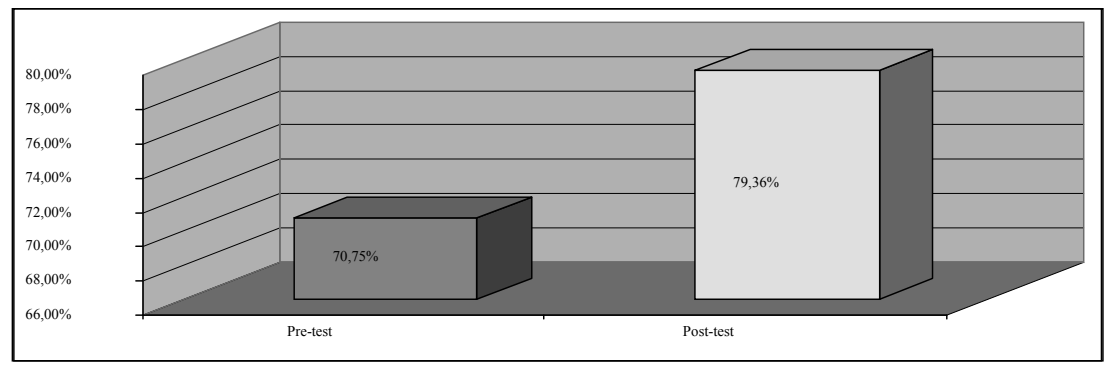

La diferencia delimitada entre el pre-test y el post-test, acorde con los puntajes porcentuales, también se puede percibir en los resultados obtenidos en cada una de las 4 habilidades lingüísticas. Los estudiantes tuvieron un mejor rendimiento en el post-test que en el pre-test: (1) en lo que corresponde a la habilidad de comprensión auditiva, la media lograda en el post-test $(15,58)$ supera la media obtenida en el pre-test $(12,83)$ en un 2,$75 ;(2)$ en cuanto a la comprensión lectora, la media alcanzada en el post-test $(15,14)$ sobrepasa la media lograda en el pre-test $(13,36)$ en un 1,78 ; (3) en lo que 
respecta a la producción oral, la media obtenida en el post-test $(24,94)$ supera la media alcanzada en el pre-test $(23,44)$ en un 1,$5 ;(4)$ referente a la habilidad de producción escrita, la media lograda en el post-test $(23,69)$ sobrepasa la media obtenida en el pre-test $(21,11)$ en un 2,58 .

En el Gráfico 4, se puede apreciar un aumento en la media porcentual obtenida en cada una de las cuatro destrezas: la comprensión auditiva (1) que es de $14 \%$ (de $64 \%$ a $78 \%$ ), seguido por la comprensión lectora y (2) la producción escrita (4) con $9 \%$ (de $67 \%$ a $76 \%$ y de $70 \%$ a $79 \%$, respectivamente), y por la producción oral (3) con 5\% (de 78\% a 83\%).

Gráfico 4: Diferencia entre rendimiento por habilidad lingüística en el pre-test y el post-test

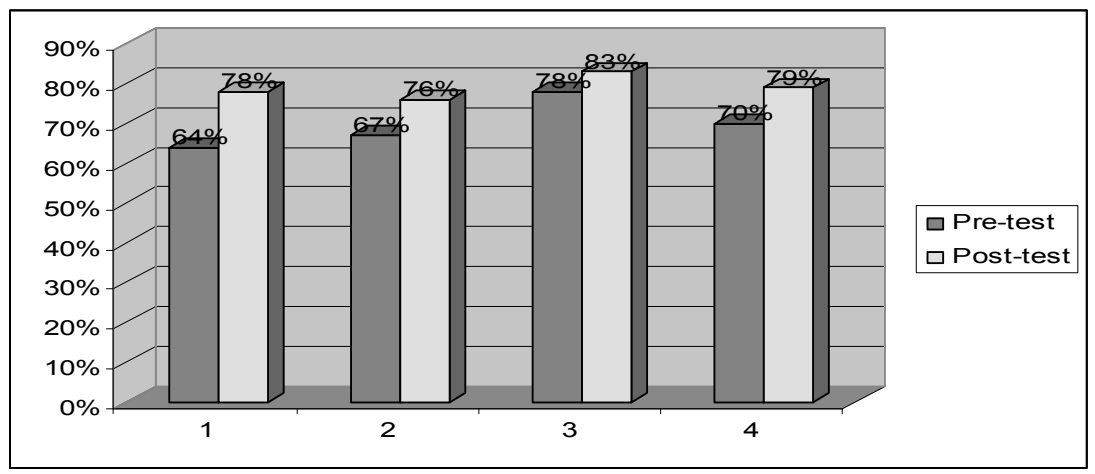

En lo concerniente a la distribución de los alumnos según el porcentaje de logro alcanzado, destaca que la tendencia en el pre-test de concentrar a los alumnos en el rango de porcentaje de $61-80 \%$ cambia y se sitúa en el posttest por sobre ésta (71-90\%). En consecuencia, los estudiantes alcanzaron un mejor rendimiento en el post-test que en el pre-test.

Para calcular el promedio de mejora porcentual en relación con los conocimientos aprendidos por los 18 estudiantes, se dividió la diferencia de los puntajes porcentuales finales $(\Sigma D)$ por el número de alumnos, lo que arroja un resultado de $8,61 \%$. Nueve estudiantes (50\% de la muestra) se sitúan por sobre este promedio; 4 alumnos $(22,2 \%$ de la muestra) se encuentran entre $6-7 \%$, y 5 estudiantes $(27,8 \%$ de la muestra) están por debajo de estos promedios.

En síntesis, de acuerdo con los resultados obtenidos a partir del pre-test y del post-test, se puede señalar que: 
- En lo concerniente a la comprensión auditiva, se observó que 12 de los 18 estudiantes tuvieron un incremento en su puntaje. Esto significa que hubo un $67 \%$ de mejoramiento en esta habilidad lingüística.

- En relación con la comprensión lectora, se vio que 13 de los 18 alumnos aumentaron su puntaje. Esto quiere decir que hubo un $\mathbf{7 2 \%}$ de mejora en esta destreza lingüística.

- En cuanto a la producción oral, se constató un incremento en el puntaje de 12 de los 18 estudiantes. Esto pone de relieve que hubo un $\mathbf{6 7 \%}$ de mejoría en esta habilidad lingüística.

- En lo referente a la producción escrita, se observó un aumento en el puntaje de 16 de los 18 alumnos. Esto revela que hubo un $\mathbf{8 9 \%}$ de mejoramiento en esta destreza lingüística.

Con respecto a los puntajes porcentuales finales logrados en las dos pruebas, se evidenció que 16 de los 18 estudiantes tuvieron un incremento en su puntaje. Esto indica que hubo un $\mathbf{8 9 \%}$ de mejoría al final del módulo de enseñanza para el español como LE.

Para determinar si el mejoramiento en el aprendizaje de determinados conocimientos por los alumnos fue estadísticamente significativo, se calculó la diferencia entre los resultados promedios del pre-test y del post-test por medio de la prueba $t$ de Student apareada. El propósito de ésta era cuantificar la diferencia entre la media de los dos tests, comprobar si ésta era significativamente distinta, y establecer objetivamente la correlación entre las variables. A fin de establecer el valor crítico se consideró un margen de error del $1 \%$, con $\mathrm{N}-1$ grados de libertad (17), el cual es igual a 2,567. Cualquier valor obtenido por sobre éste permitiría decidir sobre la efectividad de la metodología mixta utilizada.

Con respecto al análisis estadístico, se constató que dos de los valores críticos obtenidos fueron bastante altos, superando el valor crítico al $1 \%$ para prueba de una cola con $\mathrm{N}-1$ g.1 el cual es 2,567 (la comprensión auditiva $3,289 \boldsymbol{p}>\mathbf{0 . 0 1}$ y la producción escrita $4,801 \boldsymbol{p}>\mathbf{0 . 0 1}$ ). Se debe apreciar un aumento notorio en dichas destrezas lingüísticas. De acuerdo a lo anteriormente señalado, se puede validar la hipótesis planteada en esta investigación, indicando así el éxito del tratamiento, dado que los valores conseguidos son considerados estadísticamente significativos. Éstos no se pueden atribuir, de ninguna manera, ni al azar ni a la casualidad ni a los estímulos externos. Se deben al proceso de intervención llevado a cabo. 
En lo que corresponde a los valores críticos obtenidos para la comprensión lectora y la producción oral, el análisis estadístico arrojó que fueron de $2,257 \boldsymbol{p}<\mathbf{0 . 0 1}$ y $2,500 \boldsymbol{p}<\mathbf{0 . 0 1}$, respectivamente. Éstos no sobrepasaron el de 2,567. Acorde con estos datos, no se puede confirmar la hipótesis delimitada de este estudio experimental debido a que los valores no tienen validez estadística. Se debe poner en claro que este valor obtenido no significa que el experimento realizado no haya influido en éstos. El análisis del pretest ilustró que en general los puntajes promedios conseguidos estuvieron bastante buenos (comprensión lectora - 13,36 - y producción oral - 23,44). Los resultados del post-test mostraron que los puntajes promedios alcanzados habían subido (comprensión lectora - 15,14 y producción oral - 24,94). El incremento no fue significativo y por lo tanto, no se ha podido obtener un valor crítico significativo.

Se debe recalcar que al sumar los promedios de cada una de las 4 habilidades lingüísticas, para luego conseguir el puntaje porcentual total, tanto para la prueba inicial como para la prueba final, se vio claramente que el valor $t$ quedó por sobre $2,567(5,120 \boldsymbol{p}>\mathbf{0 . 0 1})$. Aquí, se debe valorar un aumento notorio en el análisis de dichos tests. Conforme a lo señalado, se puede verificar la hipótesis de investigación, poniendo en evidencia que este valor es considerado estadísticamente significativo. No cabe la menor duda de la efectividad del modelo metodológico mixto debido a los resultados logrados.

Éstos son bastante relevantes, por cuanto señalan que al enseñar en un contexto de un modelo metodológico mixto sustentado sobre la base del Enfoque de Aprendizaje Basado en Tareas y en técnicas del Enfoque Cooperativo, es posible llegar a un equilibrio tanto en la práctica como en la mejora de las distintas destrezas necesarias para el aprendizaje de conocimientos dentro de la lengua meta de los estudiantes de nivel intermedio.

Las sesiones presenciales (cara a cara) potenciaron y optimizaron aun más la enseñanza-aprendizaje de español sin dejar de lado los elementos gramaticales que se consideraron. Asimismo, se entregó una retroalimentación acorde con el objetivo de que el estudiante tuviera conciencia de sus errores e internalizara de manera más sólida los conocimientos aprendidos.

Otro elemento que se consideró esencial a fin de que los resultados de esta investigación arrojaran efectos propicios es el hecho de que en su diseño se estructuró de modo claro el espacio de instrucción presencial para la práctica de las distintas destrezas lingüísticas. De un total de 18 sesiones y media, el $100 \%$ se realizó en forma presencial, con el objetivo de que los estudiantes practicaran las diferentes habilidades acorde con contextos determinados. Además, los estudiantes contaron con un input comprensible 
y mucho más rico, a diferencia de lo que generalmente se facilitaría en la enseñanza tradicional.

Tomando en cuenta estas cifras, se puede afirmar que el aprendizaje ganado de los estudiantes del grupo experimental se debió a que la organización del diseño, la forma en que fueron presentados los materiales y las vías de entrega de estos (a través de clases presenciales, utilizando los distintos recursos de esta modalidad) estimuló que los estudiantes practicaran las habilidades de forma integrada y compacta, involucrándolos de una manera profunda de acuerdo a los objetivos del modelo metodológico mixto.

\section{A MODO DE CIERRE Y CONCLUSIÓN}

Esta investigación ha centrado su atención en contestar la pregunta en lo que corresponde a la mejora de la competencia lingüística y comunicativa a través de la práctica de las 4 habilidades lingüísticas en español como lengua extranjera en el contexto de un modelo metodológico mixto sustentado por el Enfoque por Tareas y Cooperativo.

Ahora bien, como el periodo de tratamiento fue relativamente breve ( 18 sesiones y media) y la cantidad de sujetos también pequeña (18 sujetos), se tendrá que llevar a cabo estudios posteriores para confirmar las tendencias que se han observado en este trabajo.

A lo largo del Módulo de Intervención, se pudo evidenciar la actuación y la respuesta de los alumnos frente a esta nueva metodología de enseñanza. En general, los resultados del experimento realizado pusieron en evidencia que el mejoramiento de las habilidades lingüísticas del español en estudiantes de nivel intermedio se vio altamente mejorado al aplicar un modelo de aprendizaje combinado. Esto sugiere que los estudiantes fueron apoyados en su aprendizaje de manera efectiva por el uso de la modalidad presencial, confirmándose de este modo la hipótesis.

El diseño del modelo metodológico mixto basado en tareas y en técnicas del aprendizaje cooperativo permite promover las oportunidades de interacción en la lengua meta y así lograr un mayor aprendizaje. El enfoque por tareas proveyó las bases teóricas fundamentales para la realización de las actividades en las clases presenciales. Se crearon tareas específicas con objetivos definidos y los estudiantes se situaron en el contexto en que debían trabajar, con fines de llevar a cabo cada tarea de manera positiva. La idea de introducir situaciones de comunicación real les permitió centrarse 
y reflexionar más en la lengua objeto eligiendo por tanto las estructuras idóneas para producir los textos.

Los planes de estudios basados en tareas han sido promovidos por los investigadores y educadores de la adquisición de L2 (Ellis, 2003; Willis $\&$ Willis, 2007) como una alternativa a los planes de estudios lingüísticos basándose en los siguientes principios subyacentes: (1) los planes de estudios lingüísticos no son efectivos en la promoción de la adquisición, y (2) los programas de estudios basados en tareas se construyen en función de los procesos de adquisición.

El aprendizaje de lenguas tiene la finalidad de estimular a los estudiantes no meramente con un input comprensible, sino también con tareas que requieren la negociación del significado y la participación en la comunicación significativa y natural. Todos estos principios, conceptos y criterios son compatibles con el diseño de cursos y planes de estudios basados en tareas, y pueden formar parte del esquema de trabajo en la enseñanza de la L2 y la LE.

El profesor de lenguas debe utilizar estos criterios con el propósito de diseñar cursos, planes de estudios y unidades didácticas mediante tareas. Además, él debe analizar los resultados, y corregir su práctica para añadir, modificar o eliminar algún elemento para que logre éxito y sea productivo el proceso de aprendizaje de los aprendices. Las tareas, desde muchos años, han sido parte del repertorio convencional de las técnicas didácticas de la lengua para profesores en las décadas pasadas, y lo relevante, hoy en día, se encuentra en la manera específica en que se conceptualiza una tarea. Se les recomienda a los profesores de lenguas, en su búsqueda de mejorar la calidad de su docencia, que integren en sus planes de estudios este modelo de aprendizaje con fines de mejorar sus prácticas pedagógicas.

Los elementos culturales del módulo de enseñanza giraron en torno a los hábitos alimenticios sobre todo de los chilenos. Éstos proporcionaron un valor agregado muy positivo a la ejecución de las tareas y actividades que los estudiantes tuvieron que llevar a cabo a lo largo del experimento. El hecho de encuadrar los ejercicios dentro de la cultura chilena reforzó aún más el campo lingüístico y el conocimiento de mundo de cada uno de los estudiantes. El aprendizaje de los códigos socioculturales se muestra como una ayuda con el fin de que los estudiantes sean capaces de comprender y manejar la lengua cada vez que afronten una situación comunicativa donde tengan que practicar la nueva lengua.

Existe evidencia empírica previa que demuestra que el Enfoque por Tareas y el Aprendizaje Cooperativo son centrados en el significado y son efectivos para el desarrollo y mejoramiento de la competencia lingüística y comunicativa (Lightbown 1992; Ellis 2003; Willis \& Willis 2007). No 
obstante, no existe mucha evidencia de investigaciones experimentales con el Enfoque por Tareas y el Enfoque Cooperativo en la enseñanza-aprendizaje del español como LE. Esto pone en claro que la evidencia empírica existente se relaciona con otras lenguas, entre ellas el inglés y el alemán.

En la actualidad, en Chile, una de las metodologías didácticas que se está utilizando mayoritariamente para la enseñanza de español como LE es el Enfoque Comunicativo, por lo que este estudio es un aporte importante para la didáctica de dicho idioma. En efecto, esta investigación es, primera en este país, dado que hasta la fecha no hay evidencia de que se haya experimentado con una metodología mixta para la enseñanza de español como LE/L2.

Sin lugar a dudas, este enfoque de indagación ha de enriquecer el área de enseñanza y aprendizaje de lenguas en lo que corresponde al idioma señalado. No cabe la menor duda de que tiene mucha importancia y envergadura para investigadores, educadores y profesores de lenguas. Es más, esta metodología mixta podría tener éxito en las aulas de aquellas instituciones secundarias y terciarias en los países que aún no estén utilizando estos nuevos métodos didácticos para la enseñanza de español como LE.

Por medio de este estudio fue posible llegar a conclusiones que intentan contribuir al área de la enseñanza-aprendizaje de lenguas y más específicamente en la didáctica de español como LE. Se ha podido comprobar la efectividad de un modelo metodológico mixto para la enseñanza de español como LE para efectos de mejorar la competencia lingüística y comunicativa en torno a determinados conocimientos.

Esta investigación puede formar parte de la evidencia empírica ya existente sobre la eficacia de estos enfoques metodológicos, haciendo hincapié en el hecho de que la Enseñanza-Aprendizaje de Lenguas Mediante Tareas y técnicas del Aprendizaje Cooperativo sí se concentran en el significado y en el desarrollo del conocimiento instrumental y formal del estudiante. 


\section{REFERENCIAS BIBLIOGRÁFICAS}

Bartolomé, Antonio. 1995. Algunos modelos de enseñanza para los nuevos canales. En Cabero, J. y Martínez, F. (coord.). Nuevos canales de comunicación en la enseñanza, Madrid: Centro de Estudios Ramón Areces, pp.119-141.

Breen, Michael. 1987. Learner Contributions to Task Design, en C. Candlin y D. Murphy (eds.). Language Learning Tasks. Eaglewood Cliffs, N.J: Prentice Hall International.

EluIs, RoD. 2003. Task-Based Language Learning and Teaching. Oxford: Oxford University Press.

ESTAIRE, SHEILA. 2004-2005. La enseñanza de lenguas mediante tareas: principios y planificación de unidades didácticas. Madrid: MEELE, Universidad Antonio de Nebrija.

Estaire, SheILA y JAVIER ZANÓN. 1990. El diseño de unidades didácticas para la enseñanza de una segunda lengua. Comunicación, Lenguaje y Educación, 7-8: 55-90.

1994. Planning Classwork: A Task-Based Approach. Oxford: Heinemann.

Fathman, Ann K. y Carolyn Kessler. 1993. Cooperative Language Learning in School Contexts. Annual Review of Applied Linguistics, 13: 127-140.

Germany Patricia y Anita Ferreira Cabrera. 1999. Evaluación de Competencia Comunicativa en Español como Lengua Extranjera. Concepción: Editorial Dirección de Docencia, Universidad de Concepción.

LeE, James. 2000. Tasks and Communicative Language Classrooms. Boston: McGraw-Hill.

Lightbown, Patsy. 1992. Can they do it themselves? A comprehension-based ESL course for young children, en R. Courchene, J. St. John, C. Thierren, y J. Glidden (eds.). Comprehension-based Language Teaching: Current Trends. Ottawa: University of Ottawa Press.

Moreno, Belén. (coord.). 1997. Experto en enseñanza del español como lengua extranjera: Historia de la metodología de lenguas extranjeras, con referencia al español. Madrid: Fundación Antonio de Nebrija.

Olsen, Roger y Spencer Kagan. 1992. About Cooperative Learning. En C. Kessler (ed.). Cooperative Language Learning: A Teacher's Response Book, New York: Prentice Hall, pp. 1-30.

Richards, JACk C. y Theodore S. Rodgers. 2001. Approaches and Methods in Language Teaching ( $2^{\text {nd }}$ ed.). London: Cambridge Language Teaching Library.

Roca, Julio, Marisol Valcárcel y Mercedes Verdu. 1990. Hacia un nuevo paradigma en la enseñanza de idiomas modernos: el Enfoque por Tareas. Revista Interuniversitaria de Formación de Profesorado 8: 25-46. 
SHEEn, Ron. 1994. A critical analysis of the advocacy of the task-based syllabus. TESOL Quarterly 28: 127-57.

Skehan, Peter. 1998b. A Cognitive Approach to Language Learning. Oxford: Oxford University Press.

Trujillo SÁez, Fernando. 2002. Aprendizaje Cooperativo para la enseñanza de la lengua. Publicaciones de la Facultad de Educación y Humanidades 32: 147-162.

Willis, Jane. 1996. A Framework for Task-Based Learning. Harlow: Longman.

Willis, Dave y Jane Willis. 2007. Doing Task-Based Teaching. Oxford: Oxford University Press.

ZANóN, JAVIER (coord.). 1999. La enseñanza del español mediante tareas. Madrid: Edinumen. 